\title{
Transverse Ising Chain under Periodic Instantaneous Quenches: Dynamical Many-Body Freezing and Emergence of Slow Solitary Oscillations
}

\author{
Sirshendu Bhattacharyya \\ R.R.R. Mahavidyalaya, Radhanagar, Hooghly, India \\ Arnab Das* \\ Theoretical Division (T-4), LANL, MS-B213, Los Alamos, New Mexico - 87545, USA and \\ Max-Planck Institute for the Physics of Complex Systems, \\ Nöthnitzer Str. 38, Dresden 01187, Germany (present address). \\ Subinay Dasgupta \\ Department of Physics, University of Calcutta, 92 Acharya Prafulla Chandra Road, Kolkata 700009, India
}

(Dated: November 1, 2018)

\begin{abstract}
We study the real-time dynamics of a quantum Ising chain driven periodically by instantaneous quenches of the transverse field (the transverse field varying as rectangular wave symmetric about zero). Two interesting phenomena are reported and analyzed: (i) We observe dynamical many-body freezing (DMF) [1, i.e. strongly non-monotonic freezing of the response (transverse magnetization) with respect to the driving parameters (pulse width and height) resulting from equivocal freezing behavior of all the many-body modes. The freezing occurs due to coherent suppression of dynamics of the many-body modes. For certain combination of the pulse height and period, maximal freezing (freezing peaks) are observed. For those parameter values, a massive collapse of the entire Floquet spectrum occurs. (ii) Secondly, we observe emergence of a distinct solitary oscillation with a single frequency, which can be much lower than the driving frequency. This slow oscillation, involving many high-energy modes, dominates the response remarkably in the limit of long observation time. We identify this slow oscillation as the unique survivor of destructive quantum interference between the many-body modes. The oscillation is found to decay algebraically with time to a constant value. All the key features are demonstrated analytically with numerical evaluations for specific results.
\end{abstract}

\section{INTRODUCTION}

Dynamics of driven quantum many-body system is an emerging paradigm for studying and unveiling new quantum phenomena. Last few years have witnessed a surge of theoretical endeavors in understanding dynamics of quantum many-body systems under simple drivings. A major part of these recent activities is concentrated around quantum quenches, leading to several interesting and novel issues including (but not limited to) universal quench dynamics across quantum critical points - associated quantum Kibble-Zurek mechanism, physics of non-equilibrium excitations, and the physics of thermalization in quantum systems (see for a review, Ref. 2]; and C. de Grandi et. al., S. Mondal et. al., and U. Divkaran et. al, in Ref. 3 and references therein). The main focuses of these studies, e.g., the final defect density in a quantum quench, or the effective temperature in a thermalized system, however, are insensitive to the details of the quantum coherence of the underlying many-body dynamics. For example, the dynamical idea behind quantum Kibble-Zurek mechanism [4 is a robust translation of the classical Kibble-Zurek idea [5] to quantum systems - of course, the origin of the relevant length scales and time scales are different.

Here we focus on another important class of driven quantum non-equilibrium phenomenon, where quantum coherence plays the central role. Though nonadiabaticity is a common covering for all interesting non- equilibrium phenomenon, here coherent quantum mechanical suppression of dynamics contributes crucially to the non-adiabaticity of the dynamics which makes the resulting response behavior difficult to explain using classical intuitions. We discuss dynamics of periodically driven quantum many-body system. Coherent periodic driving can give rise to surprising phenomenon in quantum many-body system, that counters our classical intuitions drastically 11. The role of quantum coherence in the important context of superfluid-insulator transition realized in periodically driven optical lattice was demonstrated earlier [6, 7]. Owing to the experimental break-through in attaining long coherence time in quantum many-body systems in last decade, for example, within the framework of atoms/ions in optical lattices and traps, this coherent regime is becoming more and more accessible experimentally (see, e.g., 8-12]). Here we study the coherent dynamics (Schrödinger dynamics at zero temperature) of a simple paradigmatic system - the transverse Ising chain 13 subjected to a train of rectangular pulses of the transverse field. Two interesting phenomenon are reported - both purely quantum mechanical in origin and are results of coherent many-body dynamics.

It has been observed recently that a class of integrable quantum many-body systems exhibit the phenomenon of dynamical many-body freezing (DMF), i.e. non-monotonic freezing behavior of all the many-body modes when driven externally by varying a parameter in the Hamiltonian continuously [1]. The said freezing 
behavior is counter-intuitive to the "classical" picture of a driven system falling out of equilibrium. The classical behavior arises from competition of two timescales: the driving period and the relaxation time of the system (see, however, [14). In contrary to the expected monotonically increasing freezing behavior of the system with respect to the driving frequency according to that picture, we observe strongly non-monotonic freezing behavior, with maximal freezing for certain combination of driving amplitude and frequency. A related phenomenon, observed in context of a single particle localized in a periodically driven potential - known as dynamical localization, or synonymously, coherent destruction of tunneling (CDT) is well studied [15 19. In [19, interestingly, it has been shown in the context of periodically driven BEC, that the driving can lead to steady BEC-like states which are different from the equilibrium ground state of the undriven Hamiltonian. Above findings motivate us investigating such phenomenon in a pulse driven manybody system, where, instead of a smoothly varying driving rate, we have sequences of instantaneous quenches and subsequent waiting times. Here we observe DMF, confirming the generality of the phenomenon beyond sinusoidal driving. We deduce the exact condition for the maximal freezing analytically and explore other characteristics of the freezing phenomenon.

In addition to DMF, we observe another interesting phenomenon away from the freezing peaks. In the limit of long observation time, we see spectacular dominance of a single long-lived oscillation (with frequency much smaller than the driving frequency) in the response dynamics. Surprisingly, this happens even in the limit of strong and fast driving (pulse amplitude and frequency much larger than the inter-spin coupling). We discuss the origin and nature of this intriguing quantum oscillation.

\section{THE MODEL AND THE DYNAMICS}

We quench the transverse field $\Gamma$ from $+\Gamma_{0}$ to $-\Gamma_{0}$ and back in successive time intervals of duration $T$ in a transverse Ising chain Hamiltonian:

$$
\mathcal{H}=-J \sum_{j=1}^{N} s_{j}^{x} s_{j+1}^{x}-\Gamma(t) \sum_{j=1}^{N} s_{j}^{z},
$$

where the field $\Gamma(t)$ varies like a square-wave with period $T$ at $t=0$ :

$$
\Gamma(t)=\left\{\begin{aligned}
& \Gamma_{0} \text { for } n T<t<\left(n+\frac{1}{2}\right) T \\
&-\Gamma_{0} \text { for }\left(n+\frac{1}{2}\right) T<t<(n+1) T
\end{aligned}\right.
$$

with $n=0,1,2, \cdots$ and $\Gamma_{0}>0$. We set the energy scale by taking $J=1$. In order to investigate the dynamics in this case, first we diagonalize Hamiltonian (1) for a given value of $\Gamma$ by Jordan-Wigner transformation followed by Fourier transform [20]. This transforms the Hamiltonian (1) into a direct-sum of Hamiltonians of non-local free fermions of momenta $k$. The Hamiltonian preserves the parity of the fermion number (even/odd) and the ground state always lies in the even-fermionic sector. We work with the projection of the Hamiltonian in this sector, given by

$$
\begin{aligned}
\mathcal{H} & =\bigoplus_{k>0} \mathcal{H}_{k} ; \\
\mathcal{H}_{k} & =(-2 i \sin k)\left[a_{k}^{\dagger} a_{-k}^{\dagger}+a_{k} a_{-k}\right] \\
& -2(\Gamma+\cos k)\left[a_{k}^{\dagger} a_{k}+a_{-k}^{\dagger} a_{-k}-1\right],
\end{aligned}
$$

where $k=(2 n+1) \pi / N ; n=0,1, \ldots, N / 2-1$. The ground state of $\mathcal{H}_{k}$ is a linear combination of the fermionic occupation number basis states $|0\rangle_{k}=\left|0_{k}, 0_{-k}\right\rangle$ (both $\pm k$ levels unoccupied) and $|1\rangle_{k}=\left|1_{k}, 1_{-k}\right\rangle$ (both $\pm k$ levels occupied), and the Hamiltonian does not couple them with the two other basis states $\left|0_{k}, 1_{-k}\right\rangle$ and $\left|1_{k}, 0_{-k}\right\rangle$. Hence starting with ground state, the dynamics always remains confined within a manifold which is the direct product of the 2-dimensional subspaces spanned by $|0\rangle_{k}$ and $|1\rangle_{k}$. We denote the eigenstates of $\mathcal{H}_{k}$ within this subspaces as $\left|(\Gamma, k)_{-}\right\rangle$(ground state), and $\left|(\Gamma, k)_{+}\right\rangle$, with eigenvalues $-\lambda(\Gamma, k), \lambda(\Gamma, k)$, where

$$
\begin{aligned}
\lambda(\Gamma, k) & =2 \sqrt{\Gamma^{2}+1+2 \Gamma \cos k} \\
\left|(\Gamma, k)_{-}\right\rangle & =i \cos \theta|1\rangle_{k}-\sin \theta|0\rangle_{k} \\
\left|(\Gamma, k)_{+}\right\rangle & =i \sin \theta|1\rangle_{k}+\cos \theta|0\rangle_{k} \\
\tan \theta & =\frac{-\sin k}{\Gamma+\cos k+\sqrt{\Gamma^{2}+1+2 \Gamma \cos k}} .
\end{aligned}
$$

We now solve the Schrödinger equation

$$
i \hbar \frac{\partial\left|\psi_{k}\right\rangle}{\partial t}=\mathcal{H}_{k}\left|\psi_{k}\right\rangle
$$

where the wave-function in the time-dependent energy eigen-basis may be expressed as

$$
\left|\psi_{k}\right\rangle=x_{-}(t)\left|(\Gamma, k)_{-}(t)\right\rangle+x_{+}(t)\left|(\Gamma, k)_{+}(t)\right\rangle .
$$

If $\Gamma(t)$ is constant (say, $\Gamma_{0}$ ) over a time interval $t_{0}$ to $t$, then we have

$$
x_{ \pm}(t)=x_{ \pm}\left(t_{0}\right) \exp \left\{\mp \frac{i}{\hbar}\left(t-t_{0}\right) \lambda\left(\Gamma_{0}, k\right)\right\} .
$$

At time $t=0$ let the system be in a state

$$
\left|\psi_{k}\right\rangle=\alpha\left|\left(\Gamma_{0}, k\right)_{-}\right\rangle+\beta\left|\left(\Gamma_{0}, k\right)_{+}\right\rangle
$$

with $|\alpha|^{2}+|\beta|^{2}=1$. Then according to Eq. 10 at $t=$ $\frac{T}{2}-\epsilon$ (where $\epsilon$ is a small positive number), the coefficients are given by,

$$
\left(\begin{array}{l}
x_{-}\left(\frac{T}{2}-\epsilon\right) \\
x_{+}\left(\frac{T}{2}-\epsilon\right)
\end{array}\right)=\left(\begin{array}{ll}
e^{i \mu_{1}} & 0 \\
0 & e^{-i \mu_{1}}
\end{array}\right)\left(\begin{array}{l}
\alpha \\
\beta
\end{array}\right),
$$

where

$$
\mu_{1}=\frac{T}{2 \hbar} \lambda\left(\Gamma_{0}, k\right)
$$


Using the continuity of $\left|\psi_{k}\right\rangle$ at $t=\frac{T}{2}$ one obtains the wave function at $t=\frac{T}{2}+\epsilon$ in terms of $\left|\left(-\Gamma_{0}, k\right)_{-}\right\rangle$and $\left|\left(-\Gamma_{0}, k\right)_{+}\right\rangle$. Time evolution in the second half proceeds in the same way as in the first half and the transformation over one full cycle is given by,

$$
\left(\begin{array}{c}
x_{-}(T+\epsilon) \\
x_{+}(T+\epsilon)
\end{array}\right)=\mathbf{U}_{k}\left(\begin{array}{c}
\alpha \\
\beta
\end{array}\right)
$$

where,

$$
\begin{aligned}
\mathbf{U}_{k} & =\left(\begin{array}{rr}
\cos \phi & -\sin \phi \\
\sin \phi & \cos \phi
\end{array}\right)\left(\begin{array}{ll}
e^{i \mu_{2}} & 0 \\
0 & e^{-i \mu_{2}}
\end{array}\right) \\
& \times\left(\begin{array}{rr}
\cos \phi & \sin \phi \\
-\sin \phi & \cos \phi
\end{array}\right)\left(\begin{array}{ll}
e^{i \mu_{1}} & 0 \\
0 & e^{-i \mu_{1}}
\end{array}\right) .
\end{aligned}
$$

Here

$$
\mu_{2}=\frac{T}{2 \hbar} \lambda\left(-\Gamma_{0}, k\right) \quad \text { and } \quad \phi=\theta_{1}-\theta_{2}
$$

where $\theta_{1}, \theta_{2}$ are the values of $\theta$ (as defined in Eq. (77) for $\Gamma=+\Gamma_{0},-\Gamma_{0}$ respectively.

During the first half-cycle after $n$ full cycles, at a time $t=n T+\tau$ with $0<\tau<\frac{T}{2}$, the coefficients are given by,

$$
\left(\begin{array}{l}
x_{-}(n T+\tau) \\
x_{+}(n T+\tau)
\end{array}\right)=\left(\begin{array}{ll}
e^{i \mu_{3}} & 0 \\
0 & e^{-i \mu_{3}}
\end{array}\right) \mathbf{U}_{k}^{n}\left(\begin{array}{l}
\alpha \\
\beta
\end{array}\right)
$$

where $\mu_{3}=\frac{\tau}{\hbar} \lambda\left(\Gamma_{0}, k\right)$. Similarly, during the second halfcycle after $n$ full cycles, at a time $t=n T+\frac{T}{2}+\tau$, the coefficients are given by,

$$
\begin{array}{r}
\left(\begin{array}{c}
x_{-}\left(n T+\frac{T}{2}+\tau\right) \\
x_{+}\left(n T+\frac{T}{2}+\tau\right)
\end{array}\right)=\left(\begin{array}{ll}
e^{i \mu_{4}} & 0 \\
0 & e^{-i \mu_{4}}
\end{array}\right) \\
\times\left(\begin{array}{rr}
\cos \phi & \sin \phi \\
-\sin \phi & \cos \phi
\end{array}\right)\left(\begin{array}{ll}
e^{i \mu_{1}} & 0 \\
0 & e^{-i \mu_{1}}
\end{array}\right) \mathbf{U}_{k}^{n}\left(\begin{array}{l}
\alpha \\
\beta
\end{array}\right)
\end{array}
$$

where $\mu_{4}=\frac{\tau}{\hbar} \lambda\left(-\Gamma_{0}, k\right)$.

Transverse magnetization $M_{z}$ (per spin) at any time is given by,

$$
M_{z}=-1+\frac{4}{N} \sum_{k=0}^{\pi} M_{k}=-1+\frac{2}{\pi} \int_{0}^{\pi} M_{k} d k
$$

where $M_{k}=\frac{1}{2}\left\langle\psi_{k}\left|\left(a_{k}^{*} a_{k}+a_{-k}^{*} a_{-k}\right)\right| \psi_{k}\right\rangle$. From Eqs. 5. 6),

$$
M_{k}=\left|\left(x_{-} \cos \theta_{j}+x_{+} \sin \theta_{j}\right)\right|^{2}
$$

with $j=1,2$ according as we are in the first or second half-cycle respectively.

In order to calculate $\mathbf{U}_{k}^{n}$, giving the time-evolution after $n$th cycle, we note that for any $2 \times 2$ matrix,

$$
\mathbf{U}_{k}^{2}=-\left(\operatorname{Tr} \mathbf{U}_{k}\right) \mathbf{1}+\left(\operatorname{det} \mathbf{U}_{k}\right) \mathbf{U}_{k}
$$

This shows that one can write

$$
\mathbf{U}_{k}^{n}=a_{n} \mathbf{1}+b_{n} \mathbf{U}_{k}
$$

The recursion relations for $a_{n}$ and $b_{n}$ can be easily solved to get

$$
a_{n}=-b_{n-1} \quad \text { and } \quad b_{n}=\sin \left(n \omega_{k}\right) / \sin \omega_{k} .
$$

where $\cos \omega_{k}=\cos \left(\mu_{1}+\mu_{2}\right) \cos ^{2} \phi+\cos \left(\mu_{1}-\mu_{2}\right) \sin ^{2} \phi$. The expressions $b_{n}$ are the Chebyshev polynomials of the second kind in $\cos \omega_{k}$.

\section{DYNAMICAL MANY-BODY FREEZING (DMF)}

The system is initially $(t=0)$ in the ground state of the Hamiltonian with $\Gamma=+\Gamma_{0}$, before it is driven by the pulses. We have computed the magnetization numerically at any time (within a cycle) by obtaining $x_{-}$and $x_{+}$from Eqs 17, 19), substituting them in Eq. 20 to get $M_{k}$ and then integrating it using Eq. (19). The result is presented in Fig. 1. Frame (a) and (b) shows that the response, i.e., the transverse magnetization $M_{z}$ which remains localized somewhere close to its initial value for all time. In other words, the response retains the memory of the breaking of the $\mathcal{Z}_{2}$ symmetry in transverse direction by the polarized initial state through all later time, though the symmetry is respected by the driving over each complete cycle. The degree of symmetry-breaking is given by the long-time average of $M_{z}$ :

$$
Q=\lim _{T_{f} \rightarrow \infty} \frac{1}{T_{f}} \int_{0}^{T_{f}} M_{z}(t) d t
$$

$Q$ is also a measure of non-adiabatic freezing - if a driving were adiabatic, the resulting response would always follow the field (i.e., trace the instantaneous ground state value of the response) and thus would preserve the symmetry of the Hamiltonian over a period. The maximum amplitude of oscillation of $M_{z}$ also determines the degree of freezing.

It is clear from Fig. 1 that for a given value of $\Gamma_{0}$, the nonadiabatic freezing $Q$ is a strongly non-monotonic function of $T$. When the condition

$$
\frac{\Gamma_{0} T}{\hbar}=\pi, 2 \pi, 3 \pi \cdots,
$$

is satisfied the freezing attains a maximum $(Q$ shows a peak), as shown in Fig. 1(b) and 1 (c). Naively speaking, for a given $\Gamma_{0}$, if $T$ is made larger, there is more time for system to react to the successive flips made, and hence the response is expected to be more adiabatic (smaller $Q$ and bigger response amplitude). This classical intuition clearly does not hold in this case, as the freezing ( $Q$ and response amplitude) is strongly non-monotonic in $T$ for a given $\Gamma_{0}$ (Fig. 1 a). Strong maximal freezing of the entire many-body system ( $Q$ peaks) observed for isolated points in the parameter space is also a surprising nonclassical feature of DMF, arising from coherent quantum dynamics [1]. 



FIG. 1: The dynamical many-body freezing (DMF) behavior of the resulting response. (a) Variation of $M_{z}$ with $t$ for different $p\left(p=\frac{\Gamma_{0} T}{\pi}\right)$ for $\Gamma_{0}=20$. (b) Variation of $Q$ with $2 \pi / T$ for $\Gamma_{0}=20$. (c) Variation of $Q$ with $p$ for different $\Gamma_{0}$. Maximal Freezing are seen for integer $p$. (d) Magnetization after 100th and 1000th cycle at different $\Gamma_{0}$ for $T=0.1$. $(\hbar=1)$

In order to derive the extremal freezing condition (24), we set $\tau=0$ and evaluate $M_{k}(t=n T)$ as a function of $n$. Thus, we are basically looking at the start of every oscillation. Also, we assume that initially (at $t=0)$ the system was in the ground state for the transverse field at that moment. Thus, we set $\alpha=1, \beta=0$ in Eq. (17), use Eq. (21) there and obtain $x_{-}(n T)$ and $x_{+}(n T)$ which is then substituted in Eq. 20]. The result is

$$
M_{k}=A_{k}+R_{k} \cos \left(2 n \omega_{k}+\delta_{k}\right)
$$

where

$$
\begin{aligned}
A_{k} & =\cos ^{2} \theta_{1}+g_{k} f_{k}, \\
R_{k}^{2} & =g_{k}^{2}\left[f_{k}^{2}+\sin ^{2}\left(2 \theta_{1}\right) \sin ^{2} \mu_{1} \sin ^{2} \omega_{k}\right], \\
\tan \delta_{k} & =\frac{1}{f_{k}} \sin \left(2 \theta_{1}\right) \sin \mu_{1} \sin \omega_{k}
\end{aligned}
$$

with

$$
\begin{aligned}
& f_{k}=\sin \left(2 \theta_{1}\right) \sin \mu_{1} \cos \omega_{k}+\sin \left(2 \theta_{2}\right) \sin \mu_{2} \\
& g_{k}=\sin (2 \phi) \sin \left(\mu_{2}\right) /\left(2 \sin ^{2} \omega_{k}\right)=\left|U_{12}\right| /\left(2 \sin ^{2} \omega_{k}\right)
\end{aligned}
$$

and

$$
\omega_{k}=\cos ^{-1}\left[\cos \left(\mu_{1}+\mu_{2}\right) \cos ^{2} \phi+\cos \left(\mu_{1}-\mu_{2}\right) \sin ^{2} \phi\right]
$$

From Eq. 20) and (25) we see, the non-adiabatic freezing parameter $Q$ (Eq. 23) is given by

$$
Q=-1+\frac{2}{\pi} \int_{0}^{\pi} A_{k} d k
$$

Now, for large $\Gamma_{0}$, from Eqs. 13$)$ and $(16)$ we get

$$
\begin{gathered}
\phi=-\frac{\pi}{2}+\frac{\sin k}{\Gamma_{0}}+\mathcal{O}\left(\frac{1}{\Gamma_{0}^{3}}\right), \quad \text { and } \\
\mu_{2}=\frac{\Gamma_{0} T}{\hbar}\left[1-\frac{\cos k}{\Gamma_{0}}+\mathcal{O}\left(\frac{1}{\Gamma_{0}^{2}}\right)\right]
\end{gathered}
$$

The off-diagonal elements of the transfer matrix $\mathbf{U}_{k}$ becomes then,

$$
\begin{aligned}
U_{12} & =i e^{-i \mu_{1}} \sin \mu_{2} \sin 2 \phi \\
& =-i \cdot e^{-i \mu_{1}}\left[\sin \left(\frac{\Gamma_{0} T}{\hbar}\right) \frac{2 \sin k}{\Gamma_{0}}+\mathcal{O}\left(1 / \Gamma_{0}^{2}\right)\right]=-U_{21}^{*}
\end{aligned}
$$

Hence, according to Eq. 16 if $\frac{\Gamma_{0} T}{\hbar}$ is an integral multiple of $\pi, \mathbf{U}_{k}$ becomes a Identity matrix up to terms $1 / \Gamma_{0}$ (since $\mu_{1} \approx-\Gamma_{0} T / \hbar$ for $\Gamma_{0} \gg 1$ ), and the system is found at the initial state (approximately) after each cycle. Note that the freezing occurs for any initial 
state, irrespective of whether it is an eigenstate of the initial Hamiltonian or not. It is also consistent with the Floquet picture of quasi-energy degeneracy (see e.g., [21, 22]) employed in explaining dynamical localization. According to Floquet theory, the above time evolution operator $\mathbf{U}_{k}$, which induces evolution from $t=0$ to $t=T$, should have the general form $\mathbf{U}_{k}=e^{i \mathbf{M}_{\mathbf{k}} t}$, where $\mathbf{M}_{\mathbf{k}}$ is a time independent Hermitian matrix (sharing same dimension and space as $\mathbf{U}_{k}$ ) 21, with eigenvectors denoted by $\left|\mu_{1 k}\right\rangle$ and $\left|\mu_{2 k}\right\rangle$ corresponding to eigenvalues $\mu_{1 k}$ and $\mu_{2 k}$, which are the Floquet quasi-energies. Now as we have shown, in our case $\mathbf{U}_{k}$ tend to the Identity matrix up to term $1 / \Gamma_{0}$ in large $\Gamma_{0}$ limit, which means both its eigenvalues $e^{i \mu_{1 k}}, e^{i \mu_{1 k}}$ tend to unity for every $k$ within the said approximation, resulting in a massive quasi-energetic degeneracy all over the many-body spectrum - the crux of DMF. Recently, another interesting manifestation of DMF is observed in periodically driven bosons in optical lattice with small amplitude driving across the tip of the Mott lobe 23 .

The mechanism of DMF with rectangular driving can be visualized appealing to the simplicity of the driving - it consists of dynamics driven by piecewise timeindependent Hamiltonians. From Eq. (32) one can see, the dynamics (in the eigen-basis $\left\{\left|\left(+\Gamma_{0}, k\right)_{ \pm}\right\rangle\right\}$of $\left.\mathcal{H}_{k}\left(+\Gamma_{0}\right)\right)$ can be broken up into successive rotation of the basis by $\phi$ (due to successive flips of the transverse field) and intermediate accumulation of phases $\mu_{1,2}$ (due to intermediate waitings of length $T / 2$ ). Clearly if one could adjust the intermediate phases $\mu_{1,2}$ such that their effect is nullified for all $k$, in each cycle, then the system would return very closely to it's initial state after every cycle for any of the eigenstates $\left\{\left|\left(+\Gamma_{0}, k\right)_{ \pm}\right\rangle\right\}$as the initial state - the eigen-state of the initial Hamiltonian becomes Floquet states with degenerate quasi-energies (albeit with a difference in sign, which does not matter in this case). This happens, as explained in the paragraph following Eq. (32), when $\Gamma_{0}$ is large and the condition for maximal freezing (Eq. 24) is satisfied. For small $\Gamma_{0}$, $\mu_{1,2}$ would retain strong $k$-dependence, and hence this massive collapse of Floquet spectrum would not have been possible (see Fig. 1 d). It is however worth noting that this simple picture of DMF cannot be extended in cases of continuously driven systems. For example, in the case of sinusoidal driving, the eigen-states of the initial Hamiltonian do not tend to return to themselves as one approaches the DMF freezing peak - they retain a strongly $k$-dependent period ( $k$ is the quasi-momentum diagonalizing the initial Hamiltonian) of oscillation which actually diverges in the thermodynamic limit for certain modes as the DMF peak is approached. Freezing in that case is visualized as vanishing of the amplitude of oscillation of each $k$ mode, rather than "all $k$-modes coming back to itself". It seems massive collapse of the entire DMF spectrum is a result of integrability of the model and simplicity of the driving.

\section{LONG-LIVED SOLITARY OSCILLATION: THE SURVIVOR OF DESTRUCTIVE INTERFERENCES}

Analysis of $M_{z}(t)$ shows that it is dominated by a distinct solitary oscillation in the long-time limit. The analysis of the response reveals sinusoidal oscillations of only two distinct timescales - one (denoted by $\omega_{0}$ ) matches with the driving period $T$ (as expected), while the other, denoted by $T_{Q}$ (corresponding to frequency $\left.\omega_{Q}=2 \pi / T_{Q}\right)$, depends on all the driving parameters. $T_{Q}$ can be much larger compared to $T$. In spite of the fact that the driving has a large amplitude and high frequency, and the system has several excitable energy levels, we observe only one distinct non-trivial frequency in the response.

This can be understood as follows. The transverse magnetization $M_{z}(t)$ (Eq. 19 at a time $t$ is a superposition of contributions $M_{k}$ for all $k$ 's (Eq. 25). For sufficiently large $n$, the argument $\left(2 n \omega_{k}+\delta_{k}\right)$ in Eq. 25p will be large (so that its cosine will fluctuate very rapidly with $k$ ) while $R_{k}$ will remain relatively slowly varying. Thus the contributions from neighboring $k$ 's will cancel out due to destructive interference (adding up with almost same amplitude but rapidly varying phase) over any small intervals of $k$, except those around the stationary points of $\omega_{k}$ (with respect to $k$ ). In the neighborhood of its stationary points, $\omega_{k}$ is expected to vary slowly with $k$, and hence the contribution from different $k^{\prime}$ s within such neighborhood is expected to add up constructively. Elsewhere the contributions adds up destructively and can hence be ignored. Thus we may write

$$
\int_{0}^{\pi} R_{k} \cos \left(2 n \omega_{k}+\delta_{k}\right) d k \approx R_{\pi / 2} \int_{\frac{\pi}{2}-\epsilon}^{\frac{\pi}{2}+\epsilon} \cos \left(2 n \omega_{k}+\delta_{\pi / 2}\right) d k
$$

By Taylor expansion of $\omega_{k}$ about the stationary point, we can write,

$$
\cos \left(2 n \omega_{k}+\delta_{\pi / 2}\right)=\cos \left(2 n \omega_{\pi / 2}+\delta_{\pi / 2}\right) \cos \left(n C\left[k-\frac{\pi}{2}\right]^{2}\right)
$$

where $C=\left(d^{2} \omega_{k} / d k^{2}\right)_{k=\pi / 2}$. This finally gives,

$$
M_{z}(n) \approx M_{0}+\frac{a}{\sqrt{n}} \cos \left(n \omega_{Q}+\delta_{\pi / 2}\right)
$$

where,

$$
\omega_{Q}=2 \omega_{\pi / 2}=2 \cos ^{-1}\left\{1-\cos ^{2} \phi\left[1-\cos \left(\mu_{1}+\mu_{2}\right)\right]\right\}
$$

and

$$
M_{0}=-1+\frac{2}{\pi} \int_{0}^{\pi} A_{k} d k, \quad a=R_{\pi / 2} \sqrt{\frac{\pi}{2 C}}
$$

Above arguments are quite generic, and variants of them can be found in different other contexts (see, e.g., [24). Survival of few such distinct oscillations of very long 

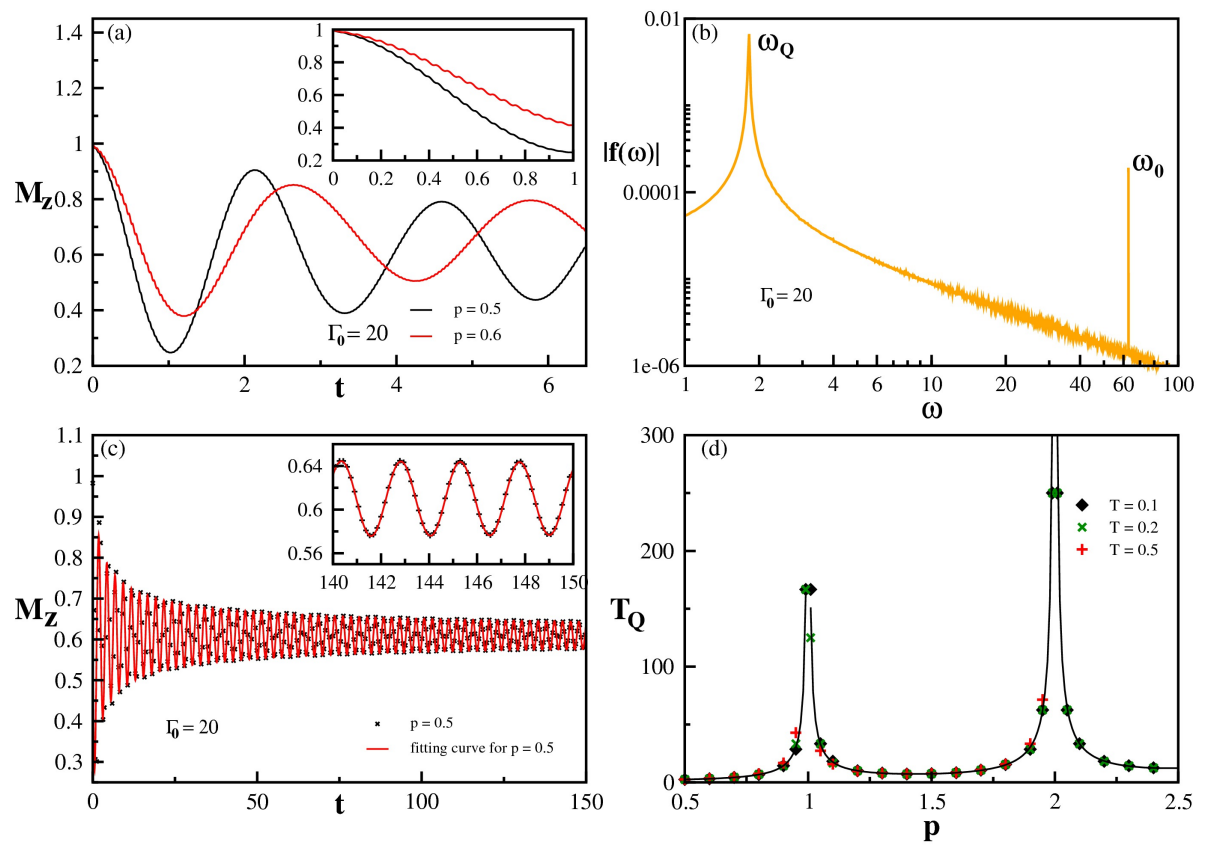

FIG. 2: Features of magnetization obtained by numerical integration of Eqs. $(25)$ and $(19)$. (a) Oscillations of two distinct time-scales are observed in $M_{z}(t)$. In addition to the expected one with period $T$ (matching to the driving), there is an additional longer time-scale prominently visible. (b) Fourier transform of $M_{z}(t)$ showing two timescales visible in (a): two distinct peaks at angular frequencies $\omega_{Q}$ and $\omega_{0}$ are observed. The peak at $\omega_{Q}=1.822$ in the Fig, matches quite well with the estimation (1.815) from Eq. [35). While $\omega_{0} \approx 2 \pi / T$, where $T=0.1$ is the driving period. (c) Long-time behavior of $M_{z}(t)$ obtained numerically (points) and that given by Eq. fitting (34) (continuous curve) using is shown. The envelop corresponding to the $1 / \sqrt{n}$ decay (Eq. 34 is visible. (d) Variation of $T_{Q}=2 \pi / \omega_{Q}$ with $p$ for $T=0.1$ obtained by Fourier transform. $T_{Q}$ tends to blow up (i.e., $\omega_{Q}$ vanishing up to order $1 / \Gamma_{0}$ ) at integer values $p$ - consistent with the observed maximal freezing at integer $p$. The points correspond to the values obtained from Fourier Transform and the continuous line is obtained from Eq. 35. $(\hbar=1)$

(compared to the driving period) time-scales was also observed in an infinite-range transverse Ising model driven periodically in time 25. The results described in this section are manifestation of more general results regarding periodically driven quantum many-body system ([26]).

We see from Eq. (35), when freezing condition ( $p=$ integer) is satisfied, $\omega_{Q}$ vanishes for large $\Gamma_{0}$ up to terms linear in $1 / \Gamma_{0}$ and $a \rightarrow 0, M_{0} \rightarrow 1$. Numerical calculation of $M_{z}$ (using Eqs. $17-20$ ) is presented in Fig 2, Discrete Fourier Transform of $M_{z}(t)$ also shows two peaks corresponding to $\omega_{0}$ and $\omega_{Q}$. The value of $\omega_{Q}$ obtained from there matches pretty well with the analytical expression in Eq. (35).

Though our results are demonstrated for rectangular pulses, similar argument can be extended for other forms of periodic drivings. The only requirements for appearance of solitary oscillations (if they exist) are certain analytical properties of the response, continuity of the spec- trum, and long driving time. Hence such oscillations are expected to appear quite generically in many-periodically driven coherent many-body quantum system, but analytical results might not be easy to extract in all cases. An extension of DMF for some other forms of periodic drivings may be achieved following [27].

The phenomena we discussed above are result of quantum coherence. Further investigations in this direction are likely to reveal many new phenomena (see. e.g. Ref.[28]). A natural open question is whether they are realizable in real experiments, in presence of the inevitable experimental imperfections existing within the present day setups. Such experimental realizations would also allow for exploring these phenomena in more generic nonintegrable systems where accurate theoretical investigations could be difficult. Experimental observation of the above phenomena might be possible within the framework of coherent quantum simulation using trapped ions and atom in optical lattice. In particular, DMF will 
have clear signature even for very small systems consisting of few spins realized in the experimental systems above, since at the freezing peaks, all the momentummodes freeze independent of system-size, whereas away from the peaks, considerable dynamics is expected for any system-size. Coherent simulation of transverse Ising Hamiltonian with time-dependent transverse field, which can be varied adiabatically, has also been realized experimentally. In layered linear Paul traps using ${ }^{171} Y b^{+}$ions [11, and ${ }^{25} \mathrm{Mg}^{+}$ions [12], they realized transverse field Ising model where they can tune both spin-spin interactions and transverse field with time.

\section{SUMMARY}

We investigate dynamics of the transverse Ising chain under periodic instantaneous quenches of the transverse field. We make two interesting observations -

(i) In the high amplitude $\left(\Gamma_{0} \gg J\right)$ and fast quenching $(T \ll J)$ limits we observe Dynamical Many-body Freezing - we see that the driven system freezes close to its initial state, and the degree of freezing is a highly nonmonotonic function of the pulse amplitude $\Gamma_{0}$ and period $T$. The extremal freezing is observed for $\Gamma_{0} T / \hbar=n \pi$ ( $n=$ positive integers). At these freezing "peaks", the system remains frozen very strongly independent of its initial state. This freezing drastically contrasts the classical notion of monotonic (with respect to the driving rate) freezing of a system under fast periodic driving a faster driving would give it lesser time to react and hence would leave it more frozen. Quantum simulation of transverse Ising chain has already been realized experimentally - the phenomenon should be amenable to experimental verification within the said set-up and similar others for quantum simulation.

(ii) In the response dynamics, we observe emergence of a single, distinct timescale $T_{Q}$ (in addition to the timescale of the driving) in the long-time limit. This distinct oscillation decays much slower than other oscillations, following a $1 / \sqrt{n}$ ( $n=$ number of sweeps) envelop. Dominance of a single non-trivial frequency in the response is surprising, since the system is driven with pulses with high (compared to the intrinsic energy scale given by the spin-spin interaction $J$ ) amplitude and frequency. We show that this surviving time-scale represents oscillations of the non-local momentum modes lying within a neighborhood of a unique point in the momentum space ( $k=\pi / 2$ here), where the contributions from the neighboring modes adds up constructively. For all other parts of the momentum space such interferences are destructive, leading to mutual cancellation of oscillations of the neighboring modes.

Acknowledgments: The authors are grateful to Andre Eckardt, Joseph Samuel and Supurna Sinha for valuable comments. AD acknowledges the support of U.S. Department of Energy through the LANL/LDRD Program. SD acknowledges financial support from CSIR (India).

*Correspondences should be addressed to AD (arnabdas@pks.mpg.de).
[1] A. Das, Phys. Rev. B 82, 172402 (2010).

[2] A. Polkovnikov, K. Sengupta, A. Silva and M. Vengalattore Rev. Mod. Phys. 83863 (2011); J. Dziarmaga, Adv. Physics 59, 1063 (2010).

[3] A. Chandra, A. Das and B. K. Chakrabarti, Quantum Quenching, Annealing and Computation, LNP 802, Springer (2010).

[4] W. H. Zurek, U. Dorner, and P. Zoller, Phys. Rev. Lett. 95, 105701 (2005); B. Damski, Phys. Rev. Lett. 95, 035701 (2005); J. Dziarmaga, Phys. Rev. Lett. 95, 245701 (2005).

[5] T. W. B. Kibble, Topology of cosmic domains and strings J. Phys. A 9, 1387 (1976); W. H. Zurek, Nature 317, 505 (1985).

[6] A. Eckardt, C. Weiss, and M. Holthaus, Phys. Rev. Lett. 95260404 (2005).

[7] A. Eckardt and M. Holthaus, EPL 80, 550004 (2007).

[8] M. Lewenstein et al., Adv. Phys 56243 (2007).

[9] I. Buluta and F. Nori, Science 326108 (2009).

[10] B. Kraus, Phys. Rev. Lett. 107, 250503 (2011).

[11] K. Kim et. al., New J. Phys. 13105003 (2011); K. Kim et. al., Nature 465590 (2010).

[12] A. Friendenauer et. al., Nat. Phys. 4, 757 (2008).

[13] B. K. Chakrabarti, A. Dutta and P. Sen (1996), Quantum
Ising Phases and Transitions in Transverse Ising Models, Springer-Verlag, Heidelberg; S. Sachdev, Quantum Phase Transition, Cambridge University Press (2001); S. Dattagupta, Paradigm Called Magnetism, World Scientific (2008).

[14] A. Eckardt and M. Holthaus, Phys. Rev. Lett. 101 245302 (2008); F. Pellegrini et. al., Phys. Rev. Lett. 107, 060401 (2011); S. Miyashita, H. De Raedt, and B. Barbara Phys. Rev. B 79104422 (2009); M. G. Bason et. al., Nature Phys. 8147 (2012).

[15] D. H. Dunlap and V. M. Kenkre, Phys. Rev. B 343625 (1986).

[16] F. Grossmann, T. Dittrich, P. Jung, and P. Hänggi Phys. Rev. Lett. 67516 (1991).

[17] M. Grifoni and P. Hänggi, Phys. Rep. 304229 (1998).

[18] A. Eckardt et. al., Phys. Rev. A 79, 013611 (2009).

[19] E. Arimondo et. al., Adv. Atomic Mol. Phys. (in press; arXiv:1203.1259 2 ).

[20] E. Lieb, T. Schultz, and D. Mattis, Ann. Phys. (N.Y.) 16, 407 (1961).

[21] A. Mostafazadeh, J. Phys A 319975 (1998).

[22] A. Eckardt and M. Holthaus, J. Phys.: Conf. Ser. 99 012007 (2008).

[23] S. Mondal, D. Pekker and K. Sengupta, 
arXiv:1204.6331v2.

[24] Y. Pomeau and P. Resibois, Phys. Rep. 19, 63 (1975).

[25] A. Das, K. Sengupta, D. Sen and B. K. Chakrabarti, Phys. Rev. B 74144423 (2006).
[26] A. Das, (unpublished).

[27] A. Sacchetti, J. Phys. A 34, 10293 (2001).

[28] A. Das and R. Moessner, arXiv:1208.0217v1 (2012). 